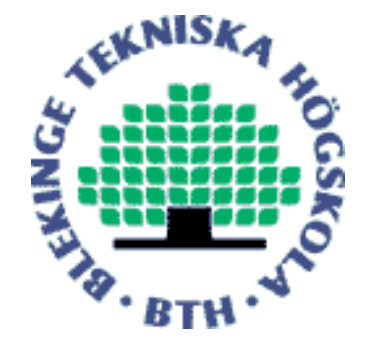

Copyright (C) 2012 IEEE.

Citation for the published paper:

SAR Imaging in Ground Plane Using Fast Backprojection for Mono- and Bistatic Cases

Viet Thuy Vu, Thomas Sjögren, Mats Pettersson

IEEE RadarCon

2012 Atlanta

This material is posted here with permission of the IEEE. Such permission of the IEEE does not in any way imply IEEE endorsement of any of BTH's products or services Internal or personal use of this material is permitted. However, permission to reprint/republish this material for advertising or promotional purposes or for creating new collective works for resale or redistribution must be obtained from the IEEE by sending a blank email message to pubs-permissions@iee.org.

By choosing to view this document, you agree to all provisions of the copyright laws protecting it. 


\title{
SAR Imaging in Ground Plane Using Fast Backprojection for Mono- and Bistatic Cases
}

\author{
Viet-Thuy Vu, Thomas K. Sjögren and Mats I. Pettersson \\ Blekinge Institute of Technology, 37179 Karlskrona, Sweden \\ Email: viet.thuy.vu@bth.se
}

\begin{abstract}
The paper discusses the possibilities to reconstruct an illuminated Synthetic Aperture Radar (SAR) scene in a ground plane instead of a slant-range plane using Fast Backprojection (FBP) algorithm. Hence, two methods to reconstruct a SAR scene in a ground plane are introduced in this paper. The methods are then considered to be extended for bistatic cases where the formation of a SAR scene in a ground plane is highly recommended. The proposals are examined with simulated SAR data and the simulation results indicate that rebuilding a SAR scene in a ground plane using FBP is possible.
\end{abstract}

\section{INTRODUCTION}

Nowadays, many geoscience and remote sensing applications are developed on Synthetic Aperture Radar (SAR). Among them, SAR imaging is considered to be one of the most crucial applications and the basis of other SAR applications. In comparison to other imaging sensors, SAR imaging is independent of weather and time of the day. In other words, a SAR system can operate in severe weather conditions such as rain, clouds, and darkness. The illuminated scene by a SAR system is then reconstructed from the measured radar echoes.

Reconstructing SAR scenes is carried out by SAR algorithms. In general, the SAR algorithms can be categorized in two groups: frequency- and time-domain. The mostly used frequency-domain algorithms are range Doppler, range migration, and chirp scaling. These algorithms are also called FFT-based algorithms as they are based on Fast Fourier Transform (FFT). The frequency-domain algorithms usually aim for minimizing processing time. This aim can lead to a number of limits such as bandwidth, integration time, motion error, either interpolation or approximation in processing, real-time processing, memory required by processing and so on which may constrain the usage of the frequency-domain algorithms. Conversely, the time-domain algorithms do not face to such the limits. This is replaced by a higher computational cost required by the time-domain algorithms. The time-domain algorithms here refer to the backprojection algorithms such as Global Backprojection (GBP) [1], Fast Backprojection (FBP) [2], and Fast Factorized Backprojection (BiFFBP) [3]. For both frequency- and time-domain algorithms, SAR scenes are usually reconstructed in a slant-range plane. However, in certain cases, SAR scenes are desired to be rebuilt in a ground plane instead of a slant-range plane. For example, reconstructing a SAR scene in ground plane is highly recommended in bistatic cases. For GBP, rebuilding a SAR scene in a slant-range plane or a ground plane has no different in terms of calculation load as well as the algorithm's performance [4]. Instead of determining the slant-range between an aperture position and an SAR image sample in a slant-range plane, the ground-range between an aperture position and an SAR image sample in a ground plane is calculated. The radar echo sample associated with this ground range is then backprojected to the considered image sample.

FBP [2] reconstructs the image of an illuminated SAR scene from radar echoes in two stages: backprojection of radar echoes into polar grids and interpolation of the data on the polar grids into a SAR image. For an $N \times N$ sample image reconstruction, FBP is shown to run roughly $\sqrt{N}$ times faster than GBP. The time-domain characteristics such as manageable motion error compensation, unlimited scene size, no limits of bandwidth and integration time, real-time processing, and optimized memory are retained with FBP. In processing, both the polar grids and the SAR image are placed in a slant-range plane. This allows simplifying the calculation in FBP as the 3D calculations are now reduced to the $2 \mathrm{D}$ calculations, i.e. calculation only in the slant-range plane. Rebuilding a SAR scene in a ground plane requires some modifications of FBP. This may make the calculations more complicated but the computational cost is supposed not to be increased significantly.

If it is possible to reconstruct a SAR scene in a ground plan using FBP, an extension of FBP to bistatic cases can be simplified significantly. Hence, the main difference between processing for monostatic and bistatic cases is only the basis for data backprojection. The back projection is performed over a spherical mapping for monostatic cases while over an ellipsoid mapping for bistatic cases.

The objective of this paper is to introduce two methods to reconstruct illuminated SAR scenes in a ground plane instead of a slant-range plane using the FBP algorithm. These methods are then considered to apply to bistatic cases. The proposal for bistatic cases is different from the results which have been published in [5]-[8] and the differences will be presented in detail. The proposals for both mono- and bistatic are finally validated and evaluated with simulated SAR data.

The rest of this paper is organized as follows: the FBP algorithm is reviewed in section 2. Two methods to reconstruct SAR scenes in a ground plane are introduced in section 3. In section 4 , the simulation results to examine the proposals are presented. The validation and limitation of the proposals are also discussed. Section 5 provides the conclusions. 


\section{FBP RECONSTRUCTING SAR SCENE IN RANGE PLANE}

In FBP [2], reconstructing illuminated SAR scenes from radar echoes is separated in two processing stages. For the backprojection stage, the full synthetic aperture is segmented into the smaller subapertures. In other words, a subaperture is created by a number of aperture positions. From the center of the $l$-th subaperture, the polar grid $(r, \theta)$ in a slantrange plane is defined. The values of $(r, \theta)$ are only in the certain ranges which ensure that the polar grids cover the SAR image as illustrated in Fig. 1. From the stationary phase condition and due to the fact that the subaperture length is small in comparison to the slant-ranges, the Nyquist sampling requirements for the polar grids are shown to be

$$
\Delta \cos \theta \leq \frac{\mathrm{c}}{2 f_{\max } d}
$$

and

$$
\Delta r \leq \frac{\mathrm{c}}{2\left(f_{\max }-f_{\min }\right)}
$$

where $\mathrm{c}$ is the speed of propagation, $f_{\max }$ and $f_{\min }$ are the maximum and minimum frequencies, respectively, and $d$ is the subaperture length. The radar echoes belonging to the $l$-th subaperture are backprojected into the polar grid over a spherical mapping. The superposition of the radar echoes in the polar coordinate $\left(r_{i}, \theta_{j}\right)$ is represented by the integral

$$
\tilde{h}_{l}\left(r_{i}, \theta_{j}\right)=\int_{t_{l}-\frac{t_{s}}{2}}^{t_{l}+\frac{t_{s}}{2}} g\left(t, \frac{R_{i, j}(t)}{\mathrm{c}}\right) d t
$$

where $t_{l}$ is the azimuth time (slow time) corresponding to the center of the $l$-th subaperture, $t_{s}$ is the integration time along the subaperture, and $g(t, \tau)$ denotes the range-compressed radar echo where $\tau$ is the range time (fast time). Without losing generality, we can assume that the linear movement of the SAR platform is expressed by

$$
\begin{aligned}
& x_{p l}(t)=v_{p l} t \\
& y_{p l}(t)=0 \\
& z_{p l}(t)=h .
\end{aligned}
$$

where $v_{p l}$ is the speed of the platform and $h$ is the flight altitude. The range $R_{i, j}$ which associates with the two-way travelling time of a radar pulse, is therefore calculated by

$$
R_{i, j}(t)=2 \sqrt{\left(v_{p l} t-r_{i} \cos \theta_{j}\right)^{2}+\left(r_{i} \sin \theta_{j}\right)^{2}}
$$

The backprojection of the radar echoes into the polar grid is applied to all subapertures. If $L$ is the number of subapertures, there will be totally $L$ polar grids whose samples are superposed by the radar echoes.

An upsampling of the data in $r$ and $\theta$ is usually required by FBP and directly affects to the final SAR image quality. There are different methods to upsample the data. For example, we can use the cubic spline data interpolation for upsampling the data in $\theta$ and the FFT/IFFT in combination with zero padding in $r$.

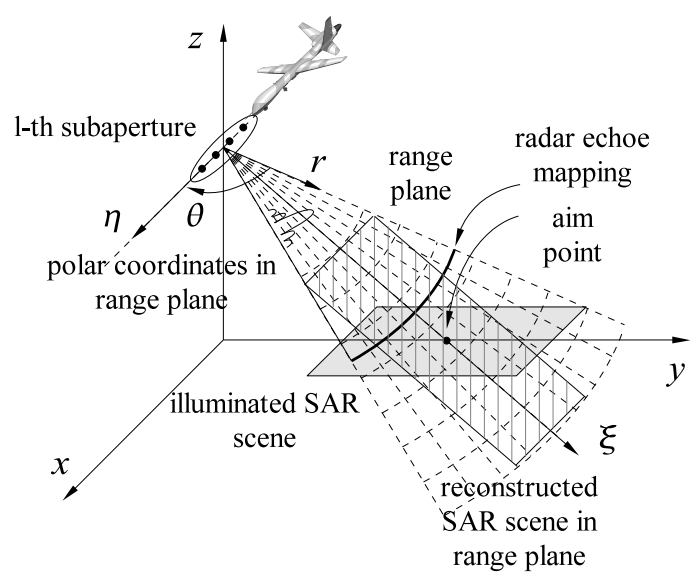

Fig. 1. Reconstructing a SAR scene in a slant-range plane. The illuminated ground scene is marked by the gray area while the reconstructed SAR image is marked by the hatched area in the slant-range plane.

For the next stage, the SAR image samples $(\eta, \xi)$ in the slant-range plane is necessary to be defined. The upsampled data on a polar grid is then interpolated into these SAR image samples forming a subaperture image. The SAR image is a coherent summing $L$ subaperture images. Thus, a SAR image sample $\left(\eta_{m}, \xi_{n}\right)$ is calculated by

$$
h_{l}\left(\eta_{m}, \xi_{n}\right)=\sum_{l=1}^{L} \tilde{h}_{l}\left(r_{m, n}, \theta_{m, n}\right)
$$

where

$$
r_{m, n}=\sqrt{\left(x_{l}-\eta_{m}\right)^{2}+\xi_{n}^{2}}
$$

and

$$
\theta_{m, n}=\operatorname{acos}\left[\frac{x_{l}-\eta_{m}}{r_{m, n}}\right]
$$

In (7) and (8), $x_{l}$ denotes the center of the $l$-th subaperture. As shown Fig. 1, the coordinates $x$ and $\eta$ are identical.

\section{ReCONSTRUCTING SAR SCENE IN GROUND PLANE}

In this section, two methods to reconstruct SAR image in a ground plane are introduced. The basic working principle of these methods and FBP is exactly the same. This means that the reconstruction of an illuminated SAR scene is also carried out in two stages: backprojection of the radar echoes into polar grids and interpolate the data on the polar grids to SAR image. The difference is only the planes where the polar grids and the SAR image are defined.

\section{A. Method 1}

In method 1, the first stage, i.e. backprojection of the radar echoes into polar grids, is identical to the first stage of FBP. The polar grids are defined in the slant-range plane. The radar echoes belonging to each subaperture are backprojected into the samples of the associated polar grid. The data on the polar grids are then upsampled in $r$ and $\theta$.

In the next stage, the Cartesian coordinates $(\eta, \xi)$ of the SAR image in a ground plane need to be determined. A 


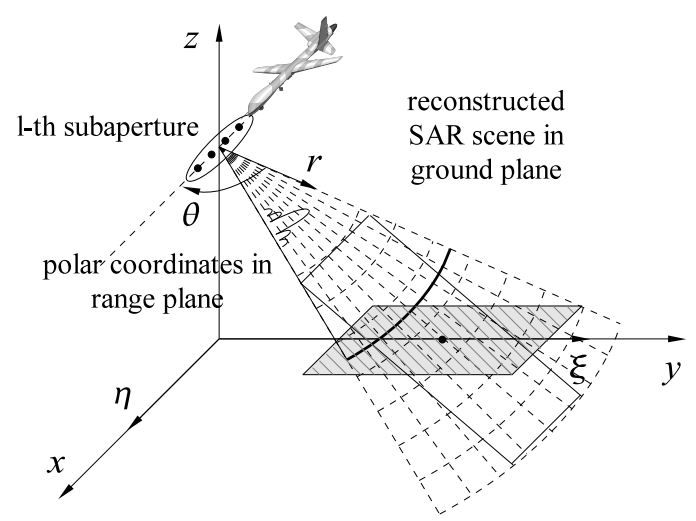

Fig. 2. Reconstructing a SAR scene in a ground plane. The polar grids are in the slant-range plane while the SAR image in the ground plane.

subaperture image in the ground plane is interpolated from the upsampled data on the corresponding polar grid. A coherent summing $L$ subaperture images results in the SAR image in the ground plane. The mathematical representation of a SAR image sample $\left(\eta_{m}, \xi_{n}\right)$ in a ground plane is also given by an equation similar to (6). However, $r_{m, n}$ and $\theta_{m, n}$ in (6) are now replaced by

$$
\breve{r}_{m, n}=2 \sqrt{\left(x_{l}-\eta_{m}\right)^{2}+\xi_{n}^{2}+h^{2}}
$$

and

$$
\breve{\theta}_{m, n}=\operatorname{acos}\left[\frac{x_{l}-\eta_{m}}{\breve{r}_{m, n}}\right]
$$

As illustrated in Fig. 2, the coordinates $(\eta, \xi)$ and $(x, y)$ in this case are the same.

\section{B. Method 2}

Instead of using the polar grids in the slant-range plane, the polar grids are proposed to be defined directly in the ground plane in the first stage as shown in Fig. 3. The Nyquist sampling requirements for the polar grids can be shown to be given by (1) and (2) based on the stationary phase conditions and the fact that the subaperture length is small in comparison to the ground ranges. The integral (3) can also be used to represent the superposition of the radar echoes on the ground polar grid $\left(r_{i}, \theta_{j}\right)$. However, the range $R_{i, j}$ in this integral is now changed to

$$
\tilde{R}_{i, j}(t)=2 \sqrt{\left(v_{p l} t-r_{i} \cos \theta_{j}\right)^{2}+\left(r_{i} \sin \theta_{j}\right)^{2}+h^{2}}
$$

Upsampling the data on the polar grids in $r$ and $\theta$ is carried out in a similar way to FBP. This means that, the cubic spline data interpolation is used for upsampling the data in $\theta$ and the FFT/IFFT in combination with zero padding for upsampling the data in $r$.

Determining the Cartesian coordinates $(\eta, \xi)$ of the SAR image in the ground plane is the first step in the next stage. Since the polar grids and the SAR image are in the same plane, i.e. ground plane, the calculations for a SAR image sample $\left(\eta_{m}, \xi_{n}\right)$ are also given by (6), (7) and (8). Please

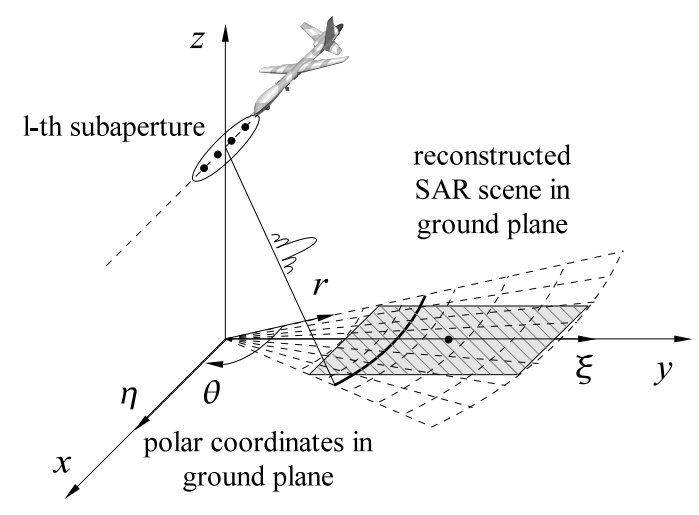

Fig. 3. Reconstructing a SAR scene in a ground plane. Both the polar grids and the SAR image are in the same ground plane.

notice that the coordinates $(\eta, \xi)$ in this case are identical to the coordinates $(x, y)$ and therefore different from the coordinates $(\eta, \xi)$ used in FBP.

\section{Apply the Method 2 to Bistatic SAR CASES}

In this part, applying the method 2 to bistatic cases is presented. This proposal is different from the Bistatic Fast Backprojection (BiFBP) and the Bistatic Fast Factorized Backprojection (BiFFBP) introduced in [5]-[7]. Hence, both BiFBP and BiFFBP work on a subimage and subaperture basis. These algorithms require beamforming instead of data backprojection into polar grids as an intermediate step in processing. The algorithm proposed in [8] clearly shows a lack of generality since the development is based on the geometry of a spaceborne-airborne bistatic SAR configuration. The elliptical range coordinate and the angular coordinate are only referenced to the transmitter trajectory.

Let us consider a bistatic SAR system with general bistatic configuration given in Fig. 4. For bistatic cases, there is no exact slant-range plane since the plane formed by the flight track of the transmitter platform and the radar pulse incident vectors are not the same as the plane formed by the flight track of receiver platform and the radar pulse scattering vectors. Reconstruction of SAR scenes in a ground plane instead of a slant-range plane is therefore highly recommended [5], [6]. As presented in the previous section, both method 1 and method 2 allow us to reconstruct SAR scene in a ground plane. These methods allow us to extend FBP to bistatic cases. To simplify calculations in the interpolation stage, method 2 will be a good choice for this extension.

As mentioned, the major difference between monostatic and bistatic SAR processing is the basis for mapping. Instead of mapping data over a spherical basis which is used in monostatic cases, backprojection of data in bistatic cases is carried out over an ellipsoidal mapping. This leads to some geometric modifications of the method 2 in order to rebuild a SAR image in a ground plane.

For bistatic cases, reconstructing an illuminated SAR scene from bistatic radar echoes is also divided into two processing 


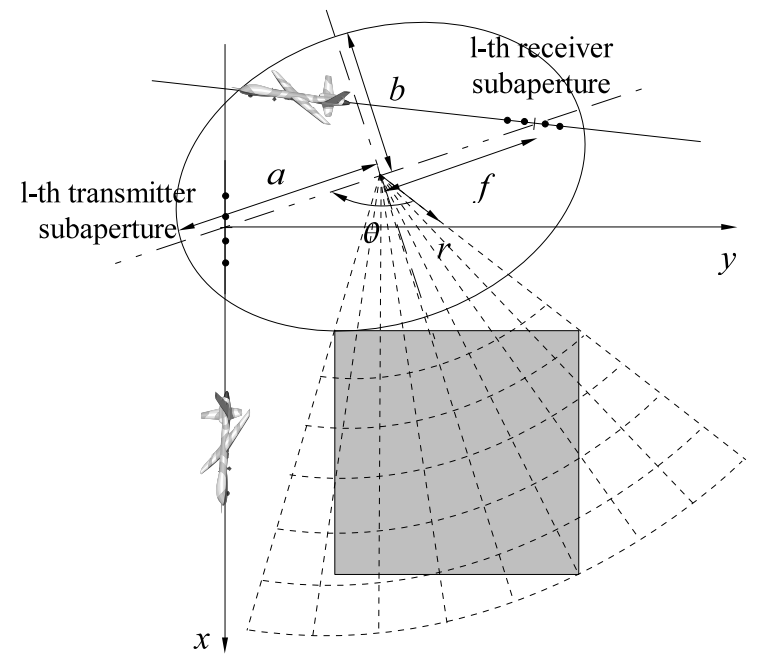

Fig. 4. Reconstructing a SAR scene in ground plane for bistatic cases.

stages. For the first stage, the full synthetic apertures, i.e. both transmitter and receiver apertures, are segmented into smaller subapertures.

The first geometric modification is the centers of polar coordinates. According to [5], the origin of the transmitter and receiver subaperture pair for beamforming is determined by the central point of the centers of those transmitter and receiver subapertures. Using the same principle, the origin of the $l$-th polar coordinates is proposed to be defined by the central point of the centers of the $l$-th transmitter subaperture and the $l$-th receiver subaperture.

From the $l$-th origin, a polar grid is defined in the ground plane. Since the backprojection of data in bistatic cases is handled over an ellipsoidal mapping, the second geometric modification, which is very natural, is to define the polar coordinates by $(r(a, b, \theta), \theta)$ where $a$ and $b$ are major and minor axes of an ellipse whose the foci are determined by the centers of the transmitter and receiver subapertures. The dependence of $r$ on $a, b$ and $\theta$ is represented by the ellipse equation showing the polar form relative to center [9]. The relationship between the linear eccentricity $f$, major axis $a$ and minor axis $b$ of an ellipse is always given by

$$
f=\sqrt{a^{2}-b^{2}}
$$

Concerning the backprojection of bistatic SAR echoes into a polar grid $(r(a, b, \theta), \theta)$, the linear eccentricity $f$ is a constant while the major axis $a$ and the minor axis $b$ will be varied for a single pair of subapertures. Since $r$ is a function of $a, b$ and $\theta$, the definition of the polar grid $(r(a, b, \theta), \theta)$ should be based on either $a$ or $b$ and $\theta$. In the case where $a$ is used for this definition, $b$ can be easily found from the relationship (12) with a known $f$ and vice versa. The coordinate $r(a, b, \theta)$ is then computed using the ellipse equation. We assume here that the Nyquist sampling requirements for the polar grids in monostatic cases, i.e. (1) and (2), are still available for bistatic cases. The derivation of the Nyquist sampling requirements for the polar grids in bistatic cases is not the topic of this paper. Hence, in the simulation, we select relative small values of $a$ or $b$ and $\Delta \theta$ to ensure that the polar grids always fulfill the Nyquist sampling requirements. The values of $(r(a, b, \theta), \theta)$ are only in the intervals so that the polar grids can cover whole the SAR image as illustrated in Fig. 4.

The mathematical expression of the backprojection of bistatic radar echoes belonging to the $l$-th subapertures into the defined polar grids $(r(a, b, \theta), \theta)$ has the similar form to (3). However, $R_{i, j}$ in the integral (3) now indicates the travelling time of a radar pulse for bistatic cases, i.e. the oneway travel time from the transmitter incident to an object on the ground scene and then scattered to the receiver.

The same upsampling methods, which have been used for the monostatic case, are also applied to bistatic cases in order to upsample the data in the $r(a, b, \theta)$ and $\theta$ directions. This means that we still use the cubic spline data interpolation for upsampling the data in $\theta$ and the FFT/IFFT in combination with zero padding in $r(a, b, \theta)$.

In the second stage, the upsampled data on each polar grid is interpolated into a subaperture image in the ground plane. Please note that, using the method 2, the Cartesian coordinates of the SAR image $(\eta, \xi)$ are identical to the coordinates $(x, y)$.

The interpolation can be seen as a transformation of the data from the polar grids to the SAR image. This transformation is the third geometric modification and based on the ellipse equation instead of (7) and (8). A coherent summing $L$ subaperture images system results in the final bistatic SAR image.

In summary, when applying the method 2 to bistatic cases, there are three major modifications which must be considered as follows:

- The centers of polar grids.

- The geometry of the polar grid $(r(a, b, \theta), \theta)$.

- The interpolation between $(r(a, b, \theta), \theta)$ and $(\eta, \xi)$.

\section{Results And Evaluations}

In this section, we provide some simulation results to validate the proposed methods to reconstruct SAR scenes in a ground plane and the application of the methods to bistatic SAR cases. Table I shows the main parameters of CARABASII [2] and LORA [3] which will be used in the simulation.

In the simulations, the ground scene is assumed to include only a number of point-like scatterers. They are equally spaced and their radar cross sections (RCS) are normalized, i.e. $\sigma=$ $1 \mathrm{~m}^{2}$. The thermal noise and the clutter are excluded from the simulated radar echoes.

\section{A. Monostatic cases}

For monostatic cases, we use only CARABAS-II's parameters given in Table I for the simulations to examine the proposed methods.

The processing parameters of the FBP algorithm have been selected as follows. The number of aperture positions in one subaperture is chosen by $L=64$ which is the optimum selection for the total number of aperture positions of 4048 
TABLE I

CARABAS-II AND LORA PARAMETERS FOR SIMULATIONS.

\begin{tabular}{ccc}
\hline \hline Parameter & CARABAS-II & LORA \\
\hline The highest frequency processed & $82.5 \mathrm{MHz}$ & \\
The lowest frequency processed & $21.9 \mathrm{MHz}$ & \\
Platform speed $v_{p l}$ & $126 \mathrm{~m} / \mathrm{s}$ & $130 \mathrm{~m} / \mathrm{s}$ \\
Aperture step & $0.9375 \mathrm{~m}$ & $0.9673 \mathrm{~m}$ \\
Flight altitude & $3700 \mathrm{~m}$ & $2900 \mathrm{~m}$ \\
Minimum range $r_{0}$ & $5900 \mathrm{~m}$ & $3000 \mathrm{~m}$ \\
PRF & $137 \mathrm{~Hz}$ & \\
Bistatic angle $\alpha$ (for bistatic case) & $60^{\circ}$ & $60^{\circ}$ \\
\hline \hline
\end{tabular}

[2]. For the monostatic cases, this selection corresponds to a subaperture length of $64 \times 0.9375=60 \mathrm{~m}$. The samples for the polar grids are selected by $\Delta r=2 \mathrm{~m}$ and $\Delta \cos \theta=0.01$ radian. These values are much smaller than the Nyquist sampling requirements given in (1) and (2). The upsampling of the data in $r$ and $\theta$ is carried out by the cubic spline data interpolation in the $\theta$ direction and the FFT/IFFT in combination with zero padding in the $r$ direction. The upsampling rates in $r$ and $\theta$ are $\epsilon_{r}=2$ and $\epsilon_{\theta}=4$, respectively.

Fig. 5 shows the simulated SAR scene reconstructed using FBP in the slant-range plane. In FBP, both the polar grids and the SAR image are in the range plane. As observed from Fig. 5, all point-like scatterers in the SAR image are well focused and appear as the point targets. We can also observe the general ultrawideband-ultrawidebeam (UWB) features of the point targets such as point-like scattering, orthogonal and non-orthogonal sidelobes.

In the next examination, method 1 is used to rebuild the SAR scene using the same SAR data. The new SAR image is shown in Fig. 6. The same processing parameters are selected for the method, i.e. $L=64, \Delta r=2 \mathrm{~m}, \Delta \cos \theta=0.01$ radian, $\epsilon_{r}=2$ and $\epsilon_{\theta}=4$. According to method 1, the polar grids are defined in the slant-range plane but the SAR image is reconstructed in the ground plane. Fig. 6 shows that all pointlike scatterers are also well focused and they also appear in the SAR image as the point targets. It is almost impossible to see any difference between the SAR images given in Fig. 5 and Fig. 6.

The simulated radar echoes are used again to examine method 2. The result is given in Fig. 7. According to this method, both polar grids and the SAR image are in the ground plane. The result shows that the focusing of all point-like scatterers is similar to the previous results. The point targets can also be observed in the SAR image given in Fig. 7. The differences between SAR images given in Fig. 5, Fig. 6 and Fig. 7 are not deteted. In other words, SAR images given in these figures are almost identical.

\section{B. Bistatic cases}

Due to the limit of the length of the paper, we cannot present here the different results corresponding to the different bistatic configurations, i.e. quasi-monostatic, azimuth-invariance and general bistatic. The general bistatic SAR configuration is selected to validate the extension of FBP to bistatic cases using method 2. In the simulation of the bistatic SAR system, the transmitter and its platform are simulated according to the CARABAS-II's parameters while the motion parameters of LORA also given in Table I are used to simulate the platform carrying a passive receiver. The flight tracks form an arbitrary angle of $60^{\circ}$.

The transmitter subaperture length has the same value as the monostatic cases but the receiver subaperture length is changed to approximately $62 \mathrm{~m}$ due to the different speeds or in other words the different aperture steps of the platforms. The angle formed by two flight tracks is $\alpha=60^{\circ}$. The samples used for the polar grids are also selected by $\Delta b=2 \mathrm{~m}$ and $\Delta \cos \theta=$ 0.01 radian. $\Delta a$ and then $\Delta r(a, b, \theta)$ can be found from the ellipse equation. The same upsampling methods and the same upsampling rates, which have been used for the monostatic cases, are utilized here.

The bistatic SAR image of the same SAR scene are given in Fig. 8. We suppose that the point-like scatters on the ground scene appear also as point targets in the bistatic SAR image since the general features of a point-like scatter illuminated by a UWB SAR system, i.e. point-like scattering, orthogonal and non-orthogonal sidelobes, can still be observed. However, the point targets are inclined with a certain angle. This inclination is believed to be dependent of the motion parameters of the platforms.

\section{Limitations}

In general, FBP and other algorithms (both time- and frequency-domain) are available for the data collected by monostatic SAR systems operating in the side-looking mode but not the forward-looking mode. For monostatic cases, methods 1 and 2 proposed in this paper are only available for the side-looking mode and do not support the forward-looking mode. However, it has been proved that the bistatic algorithms support bistatic SAR systems operating in the forward-looking mode. The extension of FBP to bistatic cases using method 2 is totally valid for bistatic forward-looking SAR without any modification. However, in some special cases of bistatic, e.g. the centers of the polar grids are very close or even identical to one of the image coordinates, the performance of the proposal for bistatic cases may be very poor since there are difficulties in defining the polar grids. These difficulties are minimized in BiFBP and BiFFBP introduced in [5], [6], respectively.

In the intermediate processing step, the proposal presented in this paper for bistatic cases uses the polar grids, i.e. working with matrices, while BiFBP and BiFFBP uses beams, i.e. working with vectors. Hence, the computational cost required by this proposal may be higher than BiFBP and BiFFBP.

\section{CONCLUSION}

In this paper, we have introduced two methods to reconstruct an illuminated SAR scene for monostatic cases in a ground plane instead of a range plane using the FBP algorithm. The backprojection of radar echoes into the polar grids in the range 


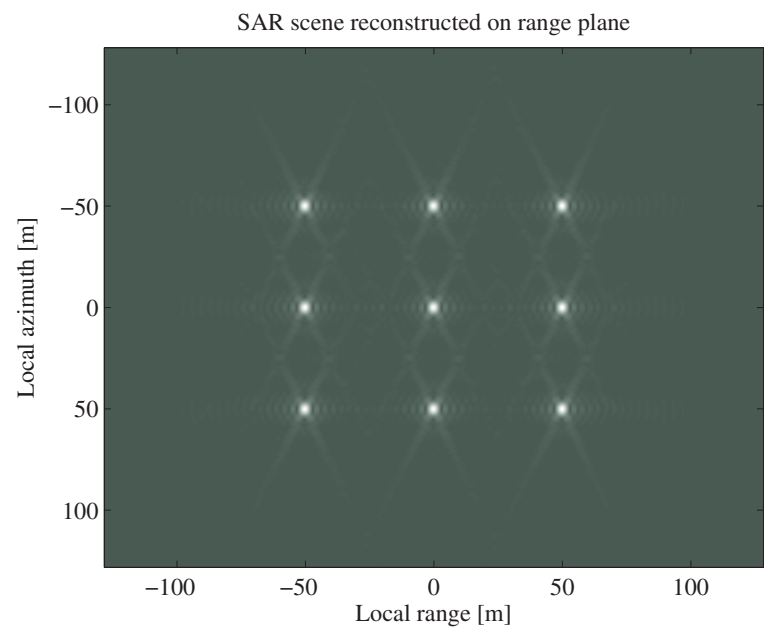

Fig. 5. The illuminated SAR scene is reconstructed with the FBP algorithm. Both polar grids and the SAR image are in the range plane.

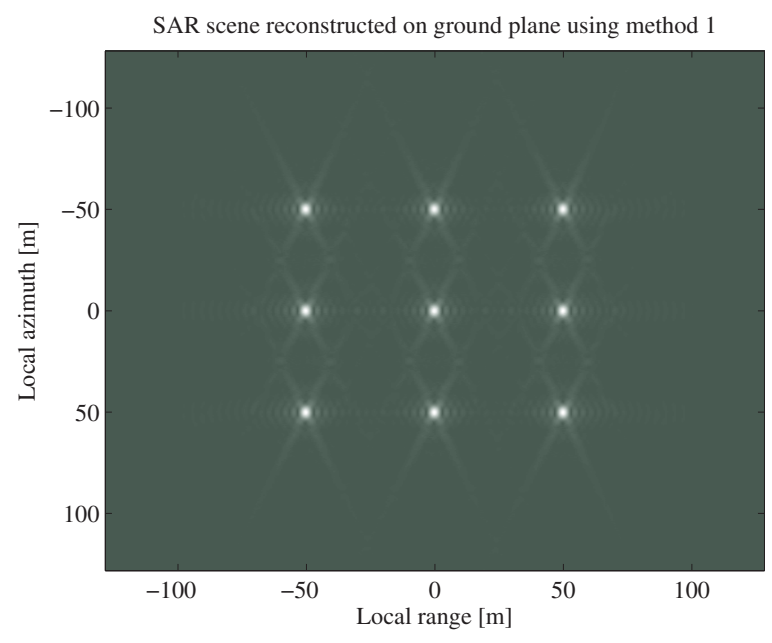

Fig. 6. The SAR scene is reconstructed using the method 1. The polar grids are in the range plane while the SAR image in the ground plane.

plane and the interpolation data from the polar grids to the SAR image in the ground plane are proposed in the method 1. In the method 2, these processing steps are performed only in the ground plane. Using method 2, the FBP algorithm is extended to bistatic cases. The proposed methods and the extension to bistatic cases have been tested successfully with the simulated monostatic and bistatic SAR data.

\section{ACKNOWLEDGMENT}

The authors would like to thank the KK-Foundation for the financial support in this research project, the Swedish Defence Research Agency, Saab Bofors Dynamics, Saab Electronic Defence Systems and RUAG Space for their cooperation.

\section{REFERENCES}

[1] L.E. Andersson, "On the determination of a function from spherical averages," SIAM Journal on Mathematical Analysis, vol. 19, no. 1, pp. 214-232, 1988.

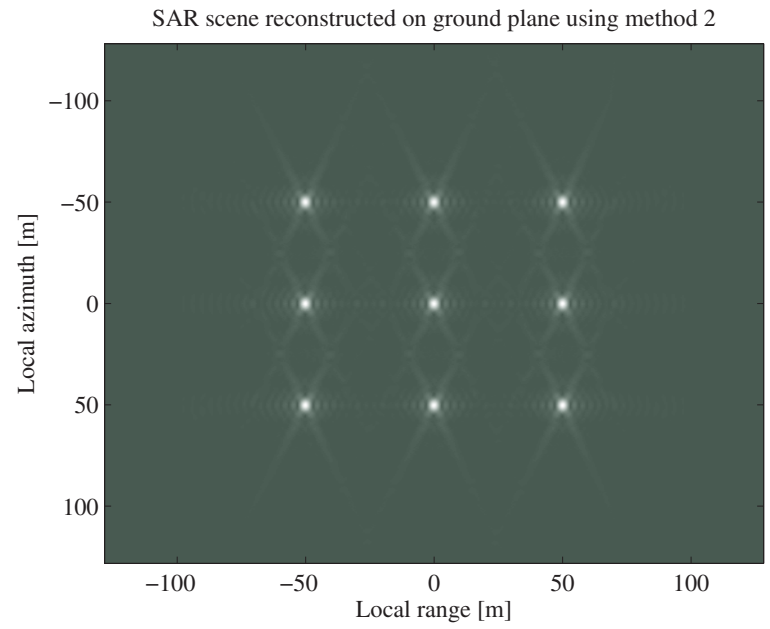

Fig. 7. The SAR scene is reconstructed using the method 2. Both polar grids and the SAR image are in the ground plane.

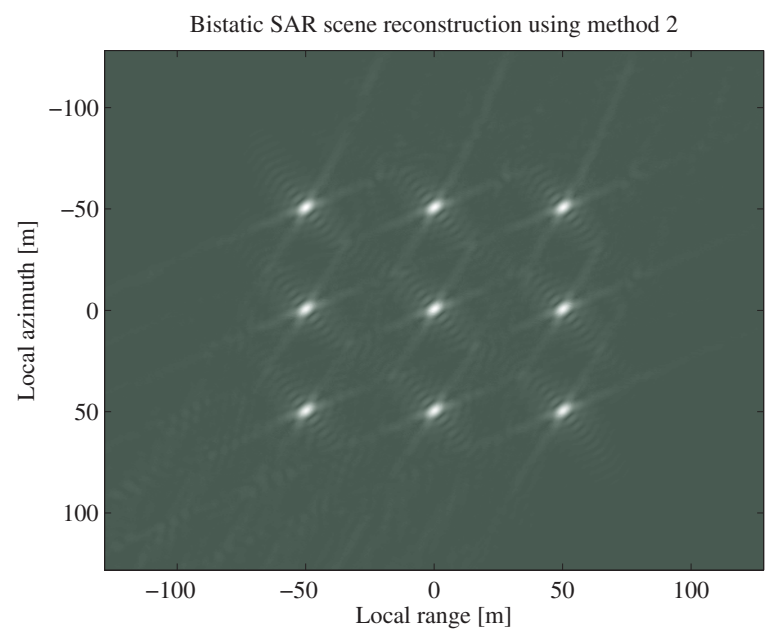

Fig. 8. The method 2 is applied to the bistatic case to reconstruct the SAR scene. Both polar grids and the SAR image are in the ground plane.

[2] A.F. Yegulalp, "Fast backprojection algorithm for synthetic aperture radar," in Proc. IEEE RadarCon'99, Waltham, MA, Apr. 1999, pp. 60-65.

[3] L.M.H. Ulander, H. Hellsten and G. Stenstrom, "Synthetic-aperture radar processing using fast factorized back-projection," IEEE Trans. Aerosp. Electron. Syst., vol. 39, no. 3, pp. 760-776, 2003.

[4] V.T. Vu, T.K. Sjögren, and M.I. Pettersson, "Phase error calculation for fast time-domain bistatic SAR algorithms," IEEE Trans. Aerosp. Electron. Syst., revised for publication.

[5] V.T. Vu, T.K. Sjögren and M.I. Pettersson, "Fast backprojection for UWB bistatic SAR," in Proc. IEEE Radar'2011, Kansas, KS, May 2011, pp. 431-434.

[6] V.T. Vu, T.K. Sjögren and M.I. Pettersson, "Fast factorized backprojection algorithm for UWB bistatic SAR image reconstruction," in Proc. IEEE IGARSS'2011, Vancouver, Canada, Jul. 2011, pp. 4237-4240.

[7] L.M.H. Ulander, P.-O. Frölind, A. Gustavsson, D. Murdin, and G. Stenström, "Fast Factorized Back-Projection for Bistatic SAR Processing," in Proc. 8th EUSAR, Aachen, Germany, June 2010, pp. 1002-1005, 2010.

[8] M. Rodriguez-Cassola, P. Prats, G. Krieger, and A. Moreira, "Efficient time-domain focussing for general bistatic SAR configurations: Bistatic fast factorised backprojection," in Proc. EUSAR'2010, Aachen, Germany, June 2010, pp. 1050-1053.

[9] L. Råde and B. Westergren, Mathematics handbook for science and engineering, 4th ed., Studentlitteratur, Lund, Sweden, 1998, pp. 81. 